\title{
Chlamydomonas reinhardtii
}

${ }^{a}$ Institute of Marine and Environmental Technology, University of Maryland Center for

6 Environmental Science, Baltimore, MD 21202, USA

$7 \quad{ }^{\mathrm{b}}$ Department of Biological Sciences, University of Maryland, Baltimore County, Baltimore, MD

821250, USA

$9{ }^{\mathrm{c}}$ Department of Marine Biotechnology, University of Maryland Baltimore County, Baltimore,

$12{ }^{1}$ Correspondence: Yantao Li 


\section{Abstract}

24 Genetic compensation through transcriptional adaptation has been proposed to explain

25 phenotypic differences between gene knockouts and knockdowns. With the rapid development of

26 reverse genetic tools such as CRISPR/Cas9 and RNAi in microalgae, it is increasingly important

27 to assess whether genetic compensation affects the phenotype of engineered algal mutants. While

28 exploring triacylglycerol (TAG) biosynthesis pathways in Chlamydomonas reinhardtii, it was

29 discovered that knockout of certain genes catalyzing rate-limiting steps of TAG biosynthesis,

30 type-2 diacylglycerol acyltransferase genes (DGTTs), triggered genetic compensation under

31 abiotic stress conditions. Genetic compensation of a DGTT1 null mutation by a related PDAT

32 gene was observed regardless of the strain background or mutagenesis approach, e.g.,

33 CRISPR/Cas 9 or insertional mutagenesis. However, no compensation was found in the PDAT

34 knockout mutant. The effect of PDAT knockout was evaluated in a $\Delta v t c 1$ mutant, in which

$35 P D A T$ was up-regulated under stress. Knockout of PDAT in the $\triangle v t c 1$ background induced a

36 12.8-fold upregulation of DGTT1 and a $272.3 \%$ increase in TAG content in $\Delta v t c 1 / p d a t 1$ cells,

37 while remaining viable. These data suggest that genetic compensation contributes to the genetic

38 robustness of microalgal TAG biosynthetic pathways, maintaining lipid and redox homeostasis

39 in the knockout mutants under abiotic stress with a mechanism distinct from that found in plants.

40 This work demonstrates examples of genetic compensation in microalgae, implies the

41 physiological relevance of genetic compensation in TAG biosynthesis, and provides guidance for

42 future genetic engineering and mutant characterization efforts. 


\section{Introduction}

Organisms have evolved various genetic buffering systems to maintain fitness in response to genetic perturbations, including functionally redundant genes (Tautz, 1992), protein feedback loops (Barabási \& Oltvai, 2004), and acquisition of adaptive mutations (Teng et al., 2013). Such buffering or compensation systems ensure similar growth or developmental outcomes despite some genetic changes (El-Brolosy \& Stainier, 2017). Genetic compensation is defined as the phenomenon whereby the effect of a deleterious mutation is buffered by the genome (Kontarakis \& Stainier, 2020). Recently, genetic compensation through transciptional adaptation, by which a homologous gene or genes are upregulated in response to an early nonsense mutation in a related gene, has been proposed as a mechanism for maintaining genetic robustness in a number of metazoans, including zebrafish, mice, and nematodes, and to explain profound differences between gene knockouts and knockdowns at certain loci (El-Brolosy et al., 2019; Z. Ma et al., 2019; Rossi et al., 2015; Serobyan et al., 2020). Likewise, comparison between gene knockouts and knockdowns in some model plant systems has revealed puzzling discrepencies that suggest transcriptional adaptation is acting to buffer the genome in these species as well (Braun et al., 2008; Chen et al., 2014; Gao et al., 2015; Rodriguez-Leal et al., 2019). microalga Chlamydomonas reinhardtii (Chlamydomonas; (Greiner et al., 2017; Jiang, Brueggeman, Horken, Plucinak, \& Weeks, 2014; Xiaobo Li et al., 2019; Molnar et al., 2009; Rohr, Sarkar, Balenger, Jeong, \& Cerutti, 2004; Schroda, 2006)), but little attention has been paid as to whether these tools might for some genes lead to very different outcomes, and more broadly, whether genetic compensation occurs in green algae (El-Brolosy \& Stainier, 2017; 
67 Kontarakis \& Stainier, 2020). Here we set out to address these questions as part of an ongoing

68 study of lipid biosynthesis in Chlamydomonas, using triacylglycerol (TAG) biosynthesis

69 pathways as examples.

Microalgae store TAG as a carbon and energy-rich compound in cells, whose synthesis can be stimulated under various stress conditions (Hu et al., 2008; Lenka et al., 2016). Nutrient deprivation such as nitrogen $(\mathrm{N})$ deprivation and phosphorus $(\mathrm{P})$ deprivation are most commonly used stressors to induce TAG production. Under $\mathrm{N}$ deprivation, algal cell growth is almost completely arrested while P deprivation promotes TAG production with moderate cell growth

75 (Iwai, Ikeda, Shimojima, \& Ohta, 2014). Biosynthesis of TAG involves sequential acylation to biosynthesis. One pathway is a de novo acyl-CoA-dependent route mediated by diacylglycerol acyltransferase (DGAT) (Ohlrogge \& Browse, 1995). Three major types of DGATs including two structurally distinctive, membrane bound type-1 and type-2 DGATs and a soluble cytosolic type-3 DGAT have been identified in plants, algae, and other eukaryotes (Cases et al., 1998;

82 TAG biosynthesis pathway is an acyl-CoA-independent route that requires membrane lipid

83 breakdown and is mediated by a plastidic membrane bound enzyme, phospholipid:diacylglycerol 84 acyltransferase (PDAT) (Dahlqvist et al., 2000; Yoon, Han, Li, Sommerfeld, \& Hu, 2012). The genome of $C$. reinhardtii harbors one type-1 DGAT gene (DGAT1), five type-2 DGAT genes (DGTT1-5), and one PDAT gene (Bagnato et al., 2017; Boyle et al., 2012; Miller et

87 al., 2010; Yoon et al., 2012). RNA-Seq analysis previously revealed that the abundance of PDAT, DGAT1 and DGTT1 transcripts was up-regulated, and that of DGTT2 and DGTT3 was moderate 
response to N deprivation (Boyle et al., 2012). Subsequent RT-qPCR analyses corroborated these

91 findings for PDAT, DGAT1 and DGTT1, while DGTT2-3 were found to be induced by N

92 deprivation (Liu, Han, Yoon, $\mathrm{Hu}, \& \mathrm{Li}, 2016$; Yoon et al., 2012). Similar results were found in

93 response to $\mathrm{P}$ deprivation, where PDAT, DGAT1, and DGTT1-4 were up-regulated during TAG

94 accumulation (Iwai et al., 2014). Functional studies using in vitro enzyme assays and gene

95 knockdowns have largely yielded results consistent with expectations based on these expression data. DGTT1-3 and PDAT were shown to catalyze TAG biosynthesis in vitro (Liu et al., 2016;

97 Yoon et al., 2012). Knockdown of DGTT1, DGTT2 or DGTT3 each resulted in a moderately

98 reduced TAG (TAG-less) phenotype under persistent stress, while knockdown of PDAT resulting

99 in a moderate TAG-less phenotype during both favorable growth and the early phase of stress

100 induction (Liu et al., 2016; Yoon et al., 2012), suggesting DGTTs and PDAT are functionally related genes involved in TAG biosynthesis in Chlamydomonas. However, in some cases,

102 analysis of gene knockdowns can be limited by off-target effects and low efficiency of gene 103 silencing (Fedorov et al., 2006; Y. Ma, Creanga, Lum, \& Beachy, 2006), so null mutants are needed to better understand gene function. Among Chlamydomonas TAG biosynthesis genes, so

105 far only pdat mutants have been characterized, which had reduced TAG content under stress

106 (Boyle et al., 2012). However, whether the PDAT insertion mutations analyzed were buffered by 107 expression of related genes such as DGTTs is not known, and up to now no DGTT mutants have been analyzed. So the precise contributions of these genes to TAG accumulation remains obscure. In this work we analyzed TAG accumulation in strains bearing null mutations in several genes including DGTT1-3 and PDAT, and found phenotypes that contradicted known

111 knockdown phenotypes in these genes. Our data showed genetic compensation through up112 regulation of related TAG biosynthetic genes for $d g t t 1$ and $d g t t 2$ mutants resulted in a higher 
113 TAG content under stress, which is contrary to the TAG-less phenotype in the respective

114 knockdown lines. Interestingly, no genetic compensation was found in the pdat mutant. However,

115 when evaluated in the $\Delta v t c 1$ background (defective for Vacuolar Transporter Chaperone 1, a

116 component of the polyphosphate polymerase complex), where PDAT expression and function is

117 likely critical for storing excess energy as TAG under stress, the PDAT knockout resulted in

118 compensation by up-regulation of DGTT1 and a higher TAG content. Our data demonstrate

119 genetic compensation exists in microalgae and imply the physiological importance of genetic

120 compensation in TAG biosynthesis under stress.

121

122 Results

123 Genetic compensation is present in microalgae

Type-2 DGATs are believed to be major contributors for TAG biosynthesis in algae, but

125 previous knockdown of Chlamydomonas type-2 DGAT genes DGTT1, DGTT2 and DGTT3

126 caused only relatively small ( 10 - 20\%) suppression of TAG accumulation (Liu et al., 2016).

127 We set out to determine whether null mutations in these genes might have larger effects, so we

128 obtained $d g t t 1, d g t t 2$ and $d g t t 3$ CLiP library knockout mutants generated by the Jonikas group

129 (Xiaobo Li et al., 2019; Xiaobo Li et al., 2016) and analyzed their TAG accumulation. Each

130 mutant strain carries a CIB1 cassette insertion that disrupts the respective DGTT gene. We

131 further examined the genotype of these mutants and found the dgtt1 mutant

132 (LMJ.RY0402.223444) insertion is in the middle of intron 1, the dgtt2 mutant

133 (LMJ.RY0402.213587) insertion is at the junction between exon 4 and intron 4, and the dgtt3

134 mutant (LMJ.RY0402.223565) insertion is at the beginning of exon 3 (Fig. 1A; Fig. S1). No 
detectable transcripts of DGTT1, DGTT2 and DGTT3 were found in the respective mutants (Fig. 1C), suggesting all the mutants are null.

TAG biosynthesis was comparatively studied in the $d g t t 1, d g t t 2$, and $d g t t 3$ mutants and their parent strain (CC5325; wild-type) under P deprivation, which was reported to induce TAG accumulation in $C$. reinhardtii while maintaining moderate cell growth (Molnar et al., 2009). Surprisingly, the $d g t t 1$ and the $d g t t 2$ knockout mutants had significantly higher TAG content than the wild-type strain (t-test, $\mathrm{p} \leq 0.05$, Fig. 1B) either on a per cell basis or per dry weight (DW) basis. The TAG content of $d g t t 1$ and $d g t t 2$ mutant cells increased by about $90 \%$ and $70 \%$ per cell, respectively, and by $45 \%$ and $76 \%$ per unit dry weight, compared with wild-type cells at $72 \mathrm{~h}$ under $\mathrm{P}$ deprivation (Fig. 1B). These results are in stark contrast to those for the knockdown strains, which had reduced TAG content (Liu et al., 2016), and they suggest a compensation mechanism exists in Chlamydomonas that affects other TAG genes/enzymes in the $d g t t 1$ and $d g t t 2$ mutant. By contrast, the $d g t t 3$ knockout mutant contained $\sim 40 \%$ less TAG per cell and per dry weight compared with the wild-type (Fig. 1B), and notably it had about twice the decrease in TAG as that caused by the $d g t t 3$ knockdown (Liu et al., 2016). In Chlamydomonas, DGTTs and PDAT are homologs. Sequence analysis revealed identities between PDAT and DGTT1, DGTT2, and DGTT3 were $50.9 \%, 49.8 \%$, and $49.2 \%$, respectively (Table S1), all above $40 \%$ sequence identity needed to induce genetic compensation by homologs in previous studies (Z. Ma et al., 2019).

To explore possible genetic compensation in the knockout mutants, the expression of TAG biosynthetic genes was examined, including that of DGTT1, DGTT2, DGTT3, PDAT, and PGD1 (encodes an MGDG-specific lipase and presumably functions with PDAT in membrane recycling) (X. Li et al., 2012; Liu et al., 2016; Yoon et al., 2012). In the dgtt1 mutant, the 
expression of $D G T T 2, D G T T 3$ and $P G D 1$ remained unchanged, but the expression of PDAT was upregulated by 9 -fold compared with the wild-type at $24 \mathrm{~h}$ under P deprivation (Fig. 1C). In the dgtt 2 mutant, the expression of all tested genes was upregulated; compared with the wild-type, the expression of DGTT1, DGTT3, PDAT and PGD1 in the $d g t t 2$ mutant was increased by 19fold, 2.6-fold, 9-fold, and 7.6-fold, respectively, at $24 \mathrm{~h}$ under P deprivation (Fig. 1C), correlating with higher TAG accumulation in the dgtt 2 mutant. In the $d g t t 3$ knockout strain, the expression level of PDAT was significantly higher than for the wild type (t-test, $\mathrm{p} \leq 0.05$; Fig. 1C), but that increase did not lead to a functional compensation for TAG accumulation (Fig. 1B). Our findings suggest that a mutation leading to a premature stop codon in two different type-2 DGAT genes likely activates a genetic compensatory network by upregulation of homologous genes in Chlamydomonas. To determine whether this genetic compensation was specific to insertional mutants in the CLiP library genetic background, or whether it might be a more general phenomenon of null mutants with premature stop codons, we used CRISPR/Cas9 to generate a dgtt1 mutant in a different strain background (C. reinhardtii CC3403). In the resulting mutant (CC-dgtt1), the DGTT1 gene carries a 1229-bp insertion corresponding to pHS_SaCas9 sequence (Cas9 expression vector) near the beginning of exon 3 of the coding region (Fig. 2A and Fig. S2A) that results in premature stop codons (Fig. S2B) and disrupts expression of the DGTTI gene. The TAG content of $C C$-dgttl was comparable to that of the wild-type on a per cell basis, and $66 \%$ higher than the wild-type per unit dry weight under $\mathrm{P}$ deprivation (Fig. 2B). Moreover, this phenotype was maintained under N deprivation (Fig. S3). Similar to the situation for the $d g t t 1$ mutant from the CLiP library, RT-qPCR analyses revealed that PDAT expression was upregulated by 6.3 -fold in the $C C$-dgtt1 mutant, while other TAG biosynthesis genes (DGTT2, DGTT3 and PGD1) remained the same as in the wild-type (Fig. 2C). 
Taken together, genetic compensation of DGTT seems to occur in Chlamydomonas mutants regardless of the strain background or early-frameshift mutant allele.

\section{Compensation of $D G T T$ knockouts is not reciprocal in the pdat mutant}

Since $C$. reinhardtii cells are capable of compensating defects of $D G T T 1$ and DGTT2 mutations by upregulating related genes such as PDAT, we asked whether the mechanism is reciprocal in the pdat mutant. To this end, we assessed TAG accumulation and the expression of the DGTT1-3 genes in a pdat knockout mutant (CC4502) previously described in (Boyle et al., 2012). Unlike the dgtt1 and dgtt2 mutants, the pdat mutant had reduced TAG levels (Fig. 3), similar to the pdat knockdown lines (Yoon et al., 2012). The relative TAG content of the pdat knockout mutant was $\sim 30 \%$ lower than that of the wild-type strain at $48 \mathrm{~h}$ and $72 \mathrm{~h}$ under $\mathrm{P}$ deprivation (Fig. 3A), similar to the decrease in TAG content previously reported for this strain in response to $\mathrm{N}$ deprivation (Boyle et al., 2012). In P-deprived pdat cells, the expression of DGTT1-3 were the same as or slightly lower than in the wild-type (Fig. 3B). These data suggest no genetic compensation of TAG biosynthesis occurs in the pdat mutant.

\section{Compensation by upregulation of DGTT1 in a $4 v t c 1 / p d a t 1$ double mutant}

Our previous work suggests $P D A T$ primarily regulates TAG biosynthesis under favorable, nutrient-replete conditions (Yoon et al., 2012) but not under persistent nutrient-deficient stress conditions (Liu et al., 2016). A possible explanation for lack of genetic compensation in the pdat mutant is thus the relatively poor function of PDAT under stress conditions, in which DGTTs are already highly expressed and responsible for TAG biosynthesis. Previous work showed P metabolism, particularly P uptake, was impaired in a $\Delta v t c l$ mutant, in which the vacuolar 
204

205

206

207

208

209

210

211

212

213

214

215

216

217

218

219

220

221

222

223

224

225

226

transport chaperon (VTC) complex was disrupted (Aksoy, Pootakham, \& Grossman, 2014;

Plouviez et al., 2021; Sanz-Luque, Saroussi, Huang, Akkawi, \& Grossman, 2020; Schmollinger et al., 2021). As such, in this $\Delta v t c 1$ mutant we reasoned that PDAT expression and function may be especially critical to recycle phospholipids and balance P metabolism under stress. To test this idea, we analyzed TAG biosynthesis in the $\triangle v t c 1$ mutant and in a VTCl-rescued strain (VTC1) under P replete and P-depleted conditions. In $\Delta v t c 1$ cells TAG content was $90 \%$ higher $(t$-test, $\mathrm{p}$ $\leq 0.05$ ) than the control under P-deprived conditions but not under P-replete conditions (Fig. 4A and B), suggesting loss of VTC1 had induced TAG biosynthesis under stress. When we analyzed the TAG biosynthetic genes in P-depleted $\Delta v t c 1$ cells, only the PDAT gene showed an increase in expression (2.8-fold; Fig. 4C) while none of the DGTT or PGD genes were upregulated, suggesting a role of PDAT in controlling TAG biosynthesis under stress in the $\Delta v t c 1$ mutant. We predicted that for the $\triangle v t c 1$ mutant, in which PDAT function is critical, if PDAT expression is interrupted, genetic compensation by other TAG biosynthetic genes may be necessary for the resulting mutant to survive under stress. To test this idea, we generated a $\Delta v t c 1 / p d a t$ double mutant by crossing the $\Delta v t c 1$ (CC5165) and the pdat (CC4502) single mutant strains. Interestingly, the TAG level in the resulting strain increased compared with the control and single mutant (Fig. 4B). After $48 \mathrm{~h}$ of $\mathrm{P}$ deprivation, the TAG content of $\Delta v t c 1 / p d a t$ cells was about $272.3 \%$ higher than the VTCl control and 95\% higher than the $\Delta v t c 1$ mutant (Fig. 4B). We found that under stress, DGTT1 transcripts were upregulated in $\Delta v t c 1 / p d a t$ cells compared with the control (by12.4-fold), but no other TAG biosynthetic genes were transcriptionally upregulated (Fig. 4C). Moreover, $\Delta v t c 1 / p d a t$ cells remained viable with moderate growth under $\mathrm{P}$ deprviation. $\Delta v t c 1 / p d a t$ cell density slightly increased from $0.69 \times 10^{6}$ cells $/ \mathrm{ml}$ to $1.01 \times 10^{6}$ cells/ml between day 0 and day 4 (Fig. S4A), while the per cell dry weight of the $\Delta v t c 1 / p d a t$ 
mutant about doubled compared with the control (VTC1) (Fig. S4B). By contrast, the growth rate and per cell dry weight of $\Delta v t c 1$ cells were similar to that of the control (Fig. S4). Therefore, our results suggest that upregulation of PDAT in the $\triangle v t c 1$ mutant results in an increase in TAG accumulation, while the effect of $P D A T$ disruption in the $\Delta v t c 1$ background is buffered by upregulation of DGTT1 in the $\Delta v t c 1 / p d a t 1$ double mutant.

\section{Discussion}

Genetic compensation, and more specifically, transcriptional adaptation through upregulation of related genes, is proposed as an underlying mechanism to explain discrepencies between knockdown and knockout phenotypes in several model systems including zebrafish and mouse (El-Brolosy et al., 2019; Z. Ma et al., 2019; Rossi et al., 2015), Caenorhabditis elegans (Serobyan et al., 2020), Arabidopsis (Braun et al., 2008; Chen et al., 2014; Gao et al., 2015), and tomato (Rodriguez-Leal et al., 2019). One mechanism for this phenomenon involves the nonsense-mediated mRNA decay pathway that is activated by early nonsense mutations (ElBrolosy et al., 2019; Z. Ma et al., 2019). However, it is not known whether this phenomenon exists across eukaryotes, including microalgae (El-Brolosy \& Stainier, 2017; Kontarakis \& Stainier, 2020). Here, we demonstrate that compensation by related genes buffers the effect of deleterious mutations in Chlamydomonas lipid biosynthesis genes, in a process that resembles transcriptional adaptation. Knockouts of DGTT1 triggered upregulation of the functionally related and homologous $P D A T$ gene regardless of the strain background, mutagenesis approach, or stress conditions (P or $\mathrm{N}$ deprivation), while knockout of DGTT2 triggered upregulation of PDAT, DGTT1 and PGD1 (Fig. 1 and Fig. 2). In the $d g t t 1, C C$-dgtt1 and dgtt2 mutant, the TAGless phenotype found in respective knockdown lines (Liu et al., 2016) was not observed. Instead, 
these mutants produced more TAG than the control, likely due to transcriptional adaptation of homologous genes. This is unlikely the case for Arabidopsis mutants defective in TAG biosynthestic genes, where knockout of DGATl and PDATl resulted in a reduced TAG phenotype in oilseeds and vegetative tissues, respectively (Fan, Yan, \& Xu, 2013; Zhang, Fan, Taylor, \& Ohlrogge, 2009). Arabidopsis DGATI and PDATl are suggested to be functionally complementary genes essential for TAG biosynthesis and normal plant development (Fan et al., 2013; Zhang et al., 2009). Whether genetic compensation contributes to the observed phenotype in Arabidopsis mutants defective in TAG biosynthetic genes requires further study.

Recent advances in reverse genetic tools including CRISPR, TALEN, and ZFN genome editing methods have greatly accelerated progress in generating knockout mutants in traditional and new algal model systems, including Chlamydomonas (Greiner et al., 2017; Jiang et al., 2014; Park, Asbury, \& Miller, 2020), Nannochloropsis (Poliner, Takeuchi, Du, Benning, \& Farré, 2018; Wang et al., 2016), Volvox carteri (Ortega-Escalante, Jasper, \& Miller, 2019), and Ectocarpus (Badis et al., 2021). Moreover, there is a genome-wide insertional mutant pool, the CLiP library (Xiaobo Li et al., 2019), for Chlamydomonas. Such tools and mutant libraries dramatically facilitate analysis of gene function. However, as we demonstrated here, genetic compensation by related genes might, in some cases, offset the effects of a null mutation, confounding genetic analysis. Likewise, previous efforts to screen and characterize TAG-less Chlamydomonas mutants from the CLiP library failed to identify any DGTT knockout mutants (XB Li, personal communication), possibly due to genetic compensation, while knockdown lines of DGTTs clearly showed a reduced-TAG phenotype (Liu et al., 2016). Therefore, when studying gene function using null mutants, preferably multiple mutant strains targeting the same gene should be obtained by a targeted genome editing method (e.g. CRISPR) or from the CLiP libarary (because 
273 some might activate genetic compensation while others might not), and these mutants should be

274 carefully evaluated for their genotypic and phenotypic traits. If genetic compensation is observed

275 in the mutants, alternative methods such as knockdown should be used to confirm the respective

276 gene function. Ideally, deletion mutants should be analyzed as they will not trigger

277 transcriptional adaptation through the nonsense-mediated mRNA decay pathway (El-Brolosy et

278 al., 2019; Z. Ma et al., 2019), though to date a relatively small number of deletion mutants have

279 been generated in Chlamydomonas due to current technical limitations.

In Chlamydomonas, biosynthesis of TAG has been implicated in the protection of the

photosynthetic electron transport chain from over-reduction during stress conditions, and TAG

essential survival role under persistent stress conditions, and second, might genetic compensation occur for other mutated TAG biosynthetic genes? To address these questions, we first examined

292 with this notion is a recent PDAT analysis in the green lineage showing that $P D A T$ plays a less 
296

297

298

299

300

301

302

303

304

305

306

307

308

309

310

311

312

313

314

315

316

317

318

TAG content by only $25 \%-30 \%$, and pdat cells had a similar growth rate as the wild type (Fig

3A) (Boyle et al., 2012).

To determine whether there might be compensation for the PDAT knockout when PDAT

function is most essential, we evaluated the effect of the PDAT knockout in a $\triangle v t c 1$ mutant, in

which PDAT expression and function are likely critical for $\Delta v t c 1$ cells to store excess energy as

TAG under stress (Fig. 4). We expected that in the absence of compensation, the knockout of

$P D A T$ in the $\Delta v t c l$ background should result in a TAG-less phenotype. Intriguingly, the

$\Delta v t c 1 / p d a t$ mutant exhibited a $272.3 \%$ higher TAG content than the VTCl control, likely because

of a large (12.8-fold) upregulation of DGTT1 (Fig. 4). The $\Delta v t c 1 / p d a t$ mutant remained viable

under stress, as its cell density and dry weight increased in response to P deprivation (Fig. S4).

So while no genetic compensation was observed in the pdat mutant, in the $\Delta v t c 1 / p d a t$ double

mutant, DGTT1 was highly overexpressed, and more TAG was produced under P deprivation.

Under stress conditions, these cells continue to capture light energy through photosynthesis, and

if electron acceptors become overreduced due to the slow growth, cytotoxic reactive oxygen

species are produced that lead to cell death (Y. Li, Sommerfeld, Chen, \& Hu, 2008). TAG

biosynthesis is likely essential for $\Delta v t c 1 / p d a t$ cells to survive during stress because TAG is the

most reduced electron sink in algae (Hu et al., 2008). In line with our findings, it was previously

reported that a Chlamydomonas mutant defective for the MGDG-specific lipase PGD1

accumulated less TAG than the wild type while its cells lost viability under stress, suggesting

TAG plays an essential role as an electron and energy sink under stress conditions, thereby

preventing generation of reactive oxygen species (Du et al., 2018; X. Li et al., 2012). Together,

these data suggest that under stress conditions, loss of TAG biosynthetic gene function can be

buffered by upregulation of a related gene or genes through transcriptional adaptation in algae. 
In summary, we have demonstrated that genetic compensation contributes to genetic robustness in microalgal TAG biosynthesis, maintaining TAG biosynthesis and redox

321 homeostasis in knockout mutants under abiotic stress. This work demonstrates, to the best of our

322 knowledge, the first reported example of genetic compensation in microalgae, implies the

323 physiological relevance of genetic compensation in TAG biosynthesis under stress, and provides

324 guidance for future genetic engineering and mutant characterization efforts. The exact

325 mechanisms inducing genetic compensation in the Chlamydomonas mutants are not understood

326 and warrant further investigation. Since transcriptional adaptation in other systems is triggered

327 by mutant mRNA decay and involves Upf3a and COMPASS components (El-Brolosy et al.,

328 2019; Z. Ma et al., 2019), testing the involvement of these components in algal genetic

329 compensation is a logical next step.

Materials and Methods

Strains and growth conditions

Chlamydomonas reinhardtii LMJ.RY0402.223444, LMJ.RY0402.213587,

LMJ.RY0402.223565 and their parent strain CC5325 (cw15; $\mathrm{mt}^{-}$) (Xiaobo Li et al., 2016),

336 CC3403 $\left(\arg 7 ; c w 15 ; \mathrm{mt}^{-}\right), \Delta v t c 1$ mutant strain CC5165 $\left(\Delta v t c 1 ; \mathrm{mt}^{-}\right)$and the VTC1 rescue strain

337 CC5166 (ars76::VTC1) (Aksoy et al., 2014) were obtained from the Chlamydomonas Resource

338 Center (http://www.chlamycollection.org). CC3403 was used as the wild-type in CRISPR/Cas9

339 mutagenesis (Greiner et al., 2017). The $\Delta v t c 1 / p d a t$ double mutant was derived from a cross

340 between CC5165 and CC4502 (methods described below). All strains were maintained in Tris-

341 acetate-phosphate (TAP) medium containing paramomycin as necessary. P deprivation was 
342 imposed by transferring $C$. reinhardtii cells to P-free TAP, in which potassium phosphate was

343 replaced by $1.5 \mathrm{mM} \mathrm{KCl}$. Ammonium chloride was omitted to generate N-free TAP. All batch

344 cultures were grown under continuous light illumination at $60 \mu \mathrm{mol}$ photons $\mathrm{m}^{-2} \mathrm{~s}^{-1}$, shaking at

$345150 \mathrm{rpm}$, room temperature.

346

347

348

349

350

\section{Gene expression measurement by RT-qPCR}

Total RNA was extracted and purified using Trizol reagent (Invitrogen) and RNeasy mini kit (Qiagen). cDNA was synthesized using Protoscript II First Strand cDNA synthesis kit (NEB). The expression of target genes was determined by RT-qPCR in Applied Biosystems 7500 using the $2^{-\Delta \Delta \mathrm{CT}}$ method normalized by the expression of $18 \mathrm{~s}$ rRNA. Details are described in the Supplemental Materials and Methods. The RT-qPCR analysis for each targeted gene was performed twice, with three biological replicates and three technical replicates for each sample. The Primer sequences used in RT-qPCR are listed in Table S2.

\section{Generation and screening of Chlamydomonas reinhardtii mutants}

The CC-dgtt1 mutant was generated by the CRISPR/Cas9 method developed by Greiner et al (Greiner et al., 2017). The parent strain of the $C C$-dgttl mutant (CC3403), the SaCas9 expression vector (pHS_SaCas9) and the sgRNA cloning vector (pCrU6.4-SaCloning-aphVIII) were obtained from the Chlamydomonas Resource Center. In brief, a customized targeting vector that expressed sgRNA specifically targeting DGTT1 was constructed in pCrU6.4-SaCloningaphVIII. The SaCas9 expression vector and the customized targeting vector that co-transformed into CC3403 by electroporation (Park et al., 2020; Serobyan et al., 2020). The transformants were selected for paromomycin resistance and screened by PCR using oligonucleotides

CrDGTT1F and CrDGTT1R (sequences in Table S2) for insertions at the DGTT1 locus. The candidates yielding PCR products larger than the expected size for the wild type DGTT1 region 
were sequenced. The detailed procedures are described in the Supplemental Materials and Methods.

The $\Delta v t c 1 / p d a t 1$ double mutant was generated by mating as described in Goodenough, 1976 (Goodenough, Hwang, \& Martin, 1976) with some modifications (procedures described in the Supplemental Materials and Methods). The crossed candidates were selected for paramomycin resistance and subsequently screened by colony PCR for a co-occurrence with the deletion of the VTC1 gene and the presence of the pdat1-1 mutation (Boyle et al., 2012) in the genome. Primers CrVTC1-pF1 and CrVTC1-stopR1 (sequences in Table S2) were used for determination of the presence (yielding a 1148 bp PCR product) or the absence (yielding no PCR products) of the VTC1 gene. Primers CrPDAT1-1-F2 and RIM5-2 (sequences in Table S2)

(Boyle et al., 2012) were used to determine the presence ( $1 \mathrm{~kb}$ PCR product) or the absence (no PCR product) of the AphIII insertion in the PDAT gene.

\section{TAG content measurement}

Total lipid from $C$. reinhardtii cells was extracted using chloroform: methanol (2:1, v/v) as previously described (Yoon et al., 2012). The lipid extracts were dried under a gentle stream of nitrogen gas and re-dissolved in chloroform. Total lipid was resolved by thin-layer chromatography (TLC) on a silica gel $60 \mathrm{~F}_{254}$ plates (EMD Millipore) using hexane: t-butyl methyl ether: acetic acid (80:20:2, v/v/v) solvent mixture as a mobile phase to develop TAG. For visualization, the developed TLC plates were sprayed with $8 \%$ (w/v) $\mathrm{H}_{3} \mathrm{PO}_{4}$ solution containing $10 \%(\mathrm{w} / \mathrm{v})$ copper (II) sulfate pentahydrate, and then charred at $180^{\circ} \mathrm{C}$ for $3 \mathrm{~min}$. The relative intensity of signals corresponding to TAG can be measured using Image J software (NIH). The absolute quantification of TAG can be calculated by the sum of fatty acid fractions composed of TAG using gas chromatography-mass spectrometry (GC-MS) as previously described (Yantao Li 
et al., 2010; Ohlrogge \& Browse, 1995). Details are described in the Supplemental Materials and Methods.

\section{Statistical analysis}

Statistical analysis was performed by Student's $t$-test with two-tailed distribution. $p$ value $\leq 0.05$ was the criteria to determine statistical significance.

\section{Acknowledgements}

This work was supported in part by grants from the U.S. National Science Foundation (CBET-1511939) and DOE Office of Fossil Energy (FE-0031914) to YL, and by NSF 1332344

to SMM. We thank Drs. William Snell, Peeyush Ranjan, and Mayanka Awasthi at University of Maryland College park for their help with generating double knockout mutants, Dr. Arthur Grossman at Carnegie Institution for Science for providing the Chlamydomonas vtcl strains used in this study, Dr. Xiaobo Li at Westlake University for discussion of unpublished mutant screening data, and Drs. Fan Zhang and Russell Hill at the Institute of Marine and Environmental Technology for their help with polyP analysis. We thank Mr. Mohamed Mahmoud-Aly at Cairo University for his help with Chlamydomonas mutant characterization.

\section{Competing financial interests}

The authors declare no competing financial interests.

\section{References}

Aksoy, M., Pootakham, W., \& Grossman, A. R. (2014). Critical Function of a Chlamydomonas reinhardtii Putative Polyphosphate Polymerase Subunit during Nutrient Deprivation. The Plant Cell, 26(10), 4214-4229. doi: 10.1105/tpc.114.129270 
Badis, Y., Scornet, D., Harada, M., Caillard, C., Godfroy, O., Raphalen, M., . . Cock, J. M. (2021). Targeted CRISPR-Cas9-based gene knockouts in the model brown alga Ectocarpus. New Phytol. doi: 10.1111/nph.17525

Bagnato, C., Prados, M. B., Franchini, G. R., Scaglia, N., Miranda, S. E., \& Beligni, M. V. (2017). Analysis of triglyceride synthesis unveils a green algal soluble diacylglycerol acyltransferase and provides clues to potential enzymatic components of the chloroplast pathway. BMC Genomics, 18(1), 223. doi: 10.1186/s12864-017-3602-0

Barabási, A. L., \& Oltvai, Z. N. (2004). Network biology: understanding the cell's functional organization. Nat Rev Genet, 5(2), 101-113. doi: 10.1038/nrg1272

Boyle, N. R., Page, M. D., Liu, B., Blaby, I. K., Casero, D., Kropat, J., . . Merchant, S. S. (2012). Three acyltransferases and nitrogen-responsive regulator are implicated in nitrogen starvation-induced triacylglycerol accumulation in Chlamydomonas. Journal of Biological Chemistry, 287(19), 15811-15825. doi: 10.1074/jbc.M111.334052

Braun, N., Wyrzykowska, J., Muller, P., David, K., Couch, D., Perrot-Rechenmann, C., \& Fleming, A. J. (2008). Conditional repression of AUXIN BINDING PROTEIN1 reveals that it coordinates cell division and cell expansion during postembryonic shoot development in Arabidopsis and tobacco. Plant Cell, 20(10), 2746-2762. doi: 10.1105/tpc.108.059048

Cases, S., Smith, S. J., Zheng, Y. W., Myers, H. M., Lear, S. R., Sande, E., . . Farese, R. V., Jr. (1998). Identification of a gene encoding an acyl CoA:diacylglycerol acyltransferase, a key enzyme in triacylglycerol synthesis. Proc Natl Acad Sci U S A, 95(22), 13018-13023. doi: 10.1073/pnas.95.22.13018

Chen, X., Grandont, L., Li, H., Hauschild, R., Paque, S., Abuzeineh, A., . . Friml, J. (2014). Inhibition of cell expansion by rapid ABP1-mediated auxin effect on microtubules. Nature, 516(7529), 90-93. doi: 10.1038/nature13889

Dahlqvist, A., Stahl, U., Lenman, M., Banas, A., Lee, M., Sandager, L., . . Stymne, S. (2000). Phospholipid:diacylglycerol acyltransferase: an enzyme that catalyzes the acyl-CoAindependent formation of triacylglycerol in yeast and plants. Proc Natl Acad Sci US A, 97(12), 6487-6492. doi: 10.1073/pnas. 120067297

Du, Z. Y., Lucker, B. F., Zienkiewicz, K., Miller, T. E., Zienkiewicz, A., Sears, B. B., . . . Benning, C. (2018). Galactoglycerolipid Lipase PGD1 Is Involved in Thylakoid Membrane Remodeling in Response to Adverse Environmental Conditions in Chlamydomonas. Plant Cell, 30(2), 447-465. doi: 10.1105/tpc.17.00446

El-Brolosy, M. A., Kontarakis, Z., Rossi, A., Kuenne, C., Günther, S., Fukuda, N., . . Stainier, D. Y. R. (2019). Genetic compensation triggered by mutant mRNA degradation. Nature, 568(7751), 193-197. doi: 10.1038/s41586-019-1064-Z

El-Brolosy, M. A., \& Stainier, D. Y. R. (2017). Genetic compensation: A phenomenon in search of mechanisms. PLoS genetics, 13(7), e1006780-e1006780. doi: 10.1371/journal.pgen.1006780

Falarz, L. J., Xu, Y., Caldo, K. M. P., Garroway, C. J., Singer, S. D., \& Chen, G. (2020). Characterization of the diversification of phospholipid:diacylglycerol acyltransferases in the green lineage. Plant J, 103(6), 2025-2038. doi: 10.1111/tpj.14880

Fan, J., Yan, C., \& Xu, C. (2013). Phospholipid:diacylglycerol acyltransferase-mediated triacylglycerol biosynthesis is crucial for protection against fatty acid-induced cell death in growing tissues of Arabidopsis. Plant J, 76(6), 930-942. doi: 10.1111/tpj.12343 
Fedorov, Y., Anderson, E. M., Birmingham, A., Reynolds, A., Karpilow, J., Robinson, K., . . . Khvorova, A. (2006). Off-target effects by siRNA can induce toxic phenotype. RNA (New York, N.Y.), 12(7), 1188-1196. doi: 10.1261/rna.28106

Gao, Y., Zhang, Y., Zhang, D., Dai, X., Estelle, M., \& Zhao, Y. (2015). Auxin binding protein 1 (ABP1) is not required for either auxin signaling or Arabidopsis development. Proc Natl Acad Sci U S A, 112(7), 2275-2280. doi: 10.1073/pnas.1500365112

Goodenough, U. W., Hwang, C., \& Martin, H. (1976). Isolation and genetic analysis of mutant strains of Chlamydomonas reinhardi defective in gametic differentiation. Genetics, 82(2), 169-186.

Greiner, A., Kelterborn, S., Evers, H., Kreimer, G., Sizova, I., \& Hegemann, P. (2017). Targeting of Photoreceptor Genes in Chlamydomonas reinhardtii via Zinc-Finger Nucleases and CRISPR/Cas9. Plant Cell, 29(10), 2498-2518. doi: 10.1105/tpc.17.00659

Hu, Q., Sommerfeld, M., Jarvis, E., Ghirardi, M., Posewitz, M., Seibert, M., \& Darzins, A. (2008). Microalgal triacylglycerols as feedstocks for biofuel production: perspectives and advances. Plant J, 54, 621-639.

Iwai, M., Ikeda, K., Shimojima, M., \& Ohta, H. (2014). Enhancement of extraplastidic oil synthesis in Chlamydomonas reinhardtii using a type-2 diacylglycerol acyltransferase with a phosphorus starvation-inducible promoter. Plant Biotechnology Journal, 12(6), 808-819. doi: 10.1111/pbi.12210

Jiang, W., Brueggeman, A. J., Horken, K. M., Plucinak, T. M., \& Weeks, D. P. (2014). Successful transient expression of Cas9/sgRNA genes in Chlamydomonas reinhardtii. Eukaryotic Cell. doi: 10.1128/ec.00213-14

Kontarakis, Z., \& Stainier, D. Y. R. (2020). Genetics in Light of Transcriptional Adaptation. Trends in Genetics, 36(12), 926-935. doi: 10.1016/j.tig.2020.08.008

Lardizabal, K. D., Mai, J. T., Wagner, N. W., Wyrick, A., Voelker, T., \& Hawkins, D. J. (2001). DGAT2 is a new diacylglycerol acyltransferase gene family: purification, cloning, and expression in insect cells of two polypeptides from Mortierella ramanniana with diacylglycerol acyltransferase activity. J Biol Chem, 276(42), 38862-38869. doi: 10.1074/jbc.M106168200

Lenka, S. K., Carbonaro, N., Park, R., Miller, S. K., Thorpe, I., \& Li, Y. T. (2016). Current advances in molecular, biochemical, and computational modeling analysis of microalgal triacylglycerol biosynthesis. Biotechnology Advances, 34, 1046-1063. doi: 10.1016/j.biotechadv.2016.06.004

Li, X., Moellering, E. R., Liu, B., Johnny, C., Fedewa, M., Sears, B. B., . . Benning, C. (2012). A galactoglycerolipid lipase is required for triacylglycerol accumulation and survival following nitrogen deprivation in Chlamydomonas reinhardtii. Plant Cell., 24. doi: 10.1105/tpc.112.105106

Li, X., Patena, W., Fauser, F., Jinkerson, R. E., Saroussi, S., Meyer, M. T., . . Jonikas, M. C. (2019). A genome-wide algal mutant library and functional screen identifies genes required for eukaryotic photosynthesis. Nature Genetics, 51(4), 627-635. doi: 10.1038/s41588-019-0370-6

Li, X., Zhang, R., Patena, W., Gang, S. S., Blum, S. R., Ivanova, N., . . Jonikas, M. C. (2016). An Indexed, Mapped Mutant Library Enables Reverse Genetics Studies of Biological Processes in Chlamydomonas reinhardtii. The Plant Cell, 28(2), 367-387. doi: 10.1105/tpc.15.00465 
Li, Y., Han, D., Hu, G., Dauvillee, D., Sommerfeld, M., Ball, S., \& Hu, Q. (2010). Chlamydomonas starchless mutant defective in ADP-glucose pyrophosphorylase hyperaccumulates triacylglycerol. Metabolic Engineering, 12, 387-391. doi: DOI: 10.1016/j.ymben.2010.02.002

Li, Y., Sommerfeld, M., Chen, F., \& Hu, Q. (2008). Consumption of oxygen by astaxanthin biosynthesis: A protective mechanism against oxidative stress in Haematococcus pluvialis (Chlorophyceae). J Plant Physiol. doi: 10.1016/j.jplph.2007.12.007

Liu, J., Han, D., Yoon, K., Hu, Q., \& Li, Y. (2016). Characterization of type 2 diacylglycerol acyltransferases in Chlamydomonas reinhardtii reveals their distinct substrate specificities and functions in triacylglycerol biosynthesis. Plant Journal, 86, 3-19. doi: 10.1111/tpj.13143

Ma, Y., Creanga, A., Lum, L., \& Beachy, P. A. (2006). Prevalence of off-target effects in Drosophila RNA interference screens. Nature, 443(7109), 359-363. doi: 10.1038/nature05179

Ma, Z., Zhu, P., Shi, H., Guo, L., Zhang, Q., Chen, Y., . . Chen, J. (2019). PTC-bearing mRNA elicits a genetic compensation response via Upf3a and COMPASS components. Nature, 568(7751), 259-263. doi: 10.1038/s41586-019-1057-y

Miller, R., Wu, G., Deshpande, R. R., Vieler, A., Gartner, K., Li, X., . . Benning, C. (2010). Changes in transcript abundance in Chlamydomonas reinhardtii following nitrogen deprivation predict diversion of metabolism. Plant Physiol, 154(4), 1737-1752. doi: 10.1104/pp.110.165159

Molnar, A., Bassett, A., Thuenemann, E., Schwach, F., Karkare, S., Ossowski, S., . . . Baulcombe, D. (2009). Highly specific gene silencing by artificial microRNAs in the unicellular alga Chlamydomonas reinhardtii. Plant Journal, 58(1), 165-174.

Oelkers, P., Cromley, D., Padamsee, M., Billheimer, J. T., \& Sturley, S. L. (2002). The DGA1 gene determines a second triglyceride synthetic pathway in yeast. J Biol Chem, 277(11), 8877-8881. doi: 10.1074/jbc.M111646200

Ohlrogge, J., \& Browse, J. (1995). Lipid biosynthesis. Plant Cell, 7(7), 957-970.

Ortega-Escalante, J. A., Jasper, R., \& Miller, S. M. (2019). CRISPR/Cas9 mutagenesis in Volvox carteri. The Plant Journal, 97(4), 661-672. doi: https://doi.org/10.1111/tpj.14149

Park, R. V., Asbury, H., \& Miller, S. M. (2020). Modification of a Chlamydomonas reinhardtii CRISPR/Cas9 transformation protocol for use with widely available electroporation equipment. MethodsX, 7, 100855. doi: https://doi.org/10.1016/j.mex.2020.100855

Plouviez, M., Fernández, E., Grossman, A. R., Sanz-Luque, E., Sells, M., Wheeler, D., \& Guieysse, B. (2021). Responses of Chlamydomonas reinhardtii during the transition from P-deficient to P-sufficient growth (the P-overplus response): The roles of the vacuolar transport chaperones and polyphosphate synthesis. Journal of Phycology, 57(3), 9881003. doi: https://doi.org/10.1111/jpy.13145

Poliner, E., Takeuchi, T., Du, Z. Y., Benning, C., \& Farré, E. M. (2018). Nontransgenic MarkerFree Gene Disruption by an Episomal CRISPR System in the Oleaginous Microalga, Nannochloropsis oceanica CCMP1779. ACS Synth Biol, 7(4), 962-968. doi: 10.1021/acssynbio.7b00362

Rodriguez-Leal, D., Xu, C., Kwon, C.-T., Soyars, C., Demesa-Arevalo, E., Man, J., ... Lippman, Z. B. (2019). Evolution of buffering in a genetic circuit controlling plant stem cell proliferation. Nature Genetics, 51(5), 786-792. doi: 10.1038/s41588-019-0389-8 
Rohr, J., Sarkar, N., Balenger, S., Jeong, B. R., \& Cerutti, H. (2004). Tandem inverted repeat system for selection of effective transgenic RNAi strains in Chlamydomonas. Plant Journal, 40(4), 611-621.

Rossi, A., Kontarakis, Z., Gerri, C., Nolte, H., Hölper, S., Krüger, M., \& Stainier, D. Y. R. (2015). Genetic compensation induced by deleterious mutations but not gene knockdowns. Nature, 524(7564), 230-233. doi: 10.1038/nature14580

Sanz-Luque, E., Saroussi, S., Huang, W., Akkawi, N., \& Grossman, A. R. (2020). Metabolic control of acclimation to nutrient deprivation dependent on polyphosphate synthesis. Sci $A d v, 6(40)$. doi: 10.1126/sciadv.abb5351

Schmollinger, S., Chen, S., Strenkert, D., Hui, C., Ralle, M., \& Merchant, S. S. (2021). Singlecell visualization and quantification of trace metals in Chlamydomonas lysosome-related organelles. Proc Natl Acad Sci U S A, 118(16). doi: 10.1073/pnas.2026811118

Schroda, M. (2006). RNA silencing in Chlamydomonas: mechanisms and tools. Current Genetics, 49(2), 69-84. doi: 10.1007/s00294-005-0042-1

Serobyan, V., Kontarakis, Z., El-Brolosy, M. A., Welker, J. M., Tolstenkov, O., Saadeldein, A. M., . . . Stainier, D. Y. R. (2020). Transcriptional adaptation in Caenorhabditis elegans. eLife, 9, e50014. doi: 10.7554/eLife.50014

Tautz, D. (1992). Redundancies, development and the flow of information. Bioessays, 14(4), 263-266. doi: 10.1002/bies.950140410

Teng, X., Dayhoff-Brannigan, M., Cheng, W.-C., Gilbert, Catherine E., Sing, Cierra N., Diny, Nicola L., . . . Hardwick, J. M. (2013). Genome-wide Consequences of Deleting Any Single Gene. Molecular Cell, 52(4), 485-494. doi: https://doi.org/10.1016/j.molcel.2013.09.026

Wang, Q., Lu, Y., Xin, Y., Wei, L., Huang, S., \& Xu, J. (2016). Genome editing of model oleaginous microalgae Nannochloropsis spp. by CRISPR/Cas9. Plant J, 88. doi: $10.1111 /$ tpj. 13307

Yoon, K., Han, D., Li, Y., Sommerfeld, M., \& Hu, Q. (2012). Phospholipid:diacylglycerol acyltransferase is a multifunctional enzyme involved in membrane lipid turnover and degradation while synthesizing triacylglycerol in the unicellular green microalga Chlamydomonas reinhardtii. Plant Cell, 24, 3708-3724. doi: 10.1105/tpc.112.100701

Zhang, M., Fan, J., Taylor, D. C., \& Ohlrogge, J. B. (2009). DGAT1 and PDAT1 acyltransferases have overlapping functions in Arabidopsis triacylglycerol biosynthesis and are essential for normal pollen and seed development. The Plant Cell, 21(12), 38853901. doi: 10.1105/tpc.109.071795 
A
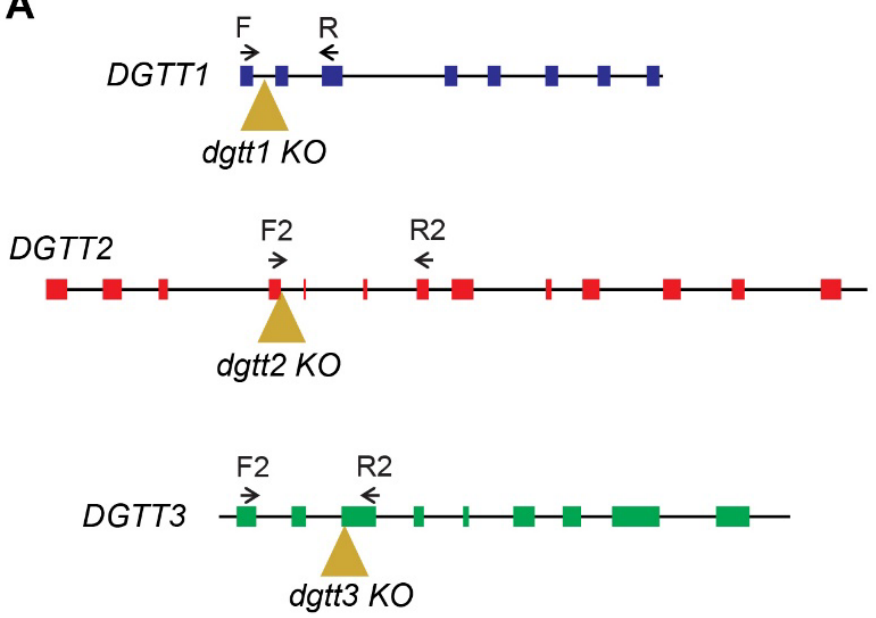

C
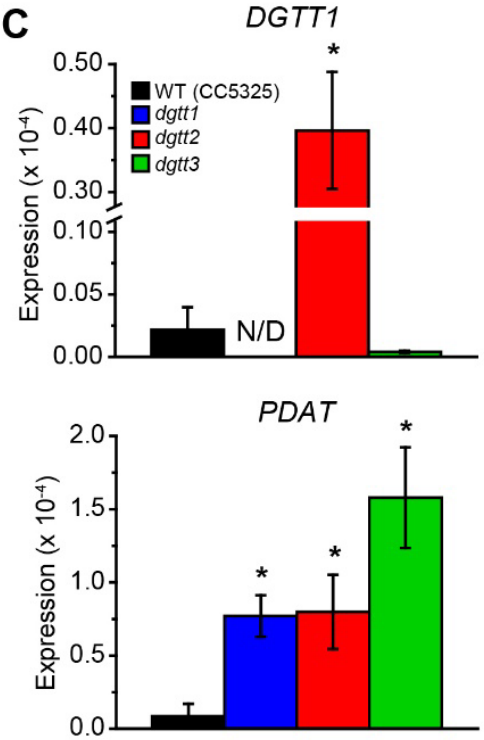

B

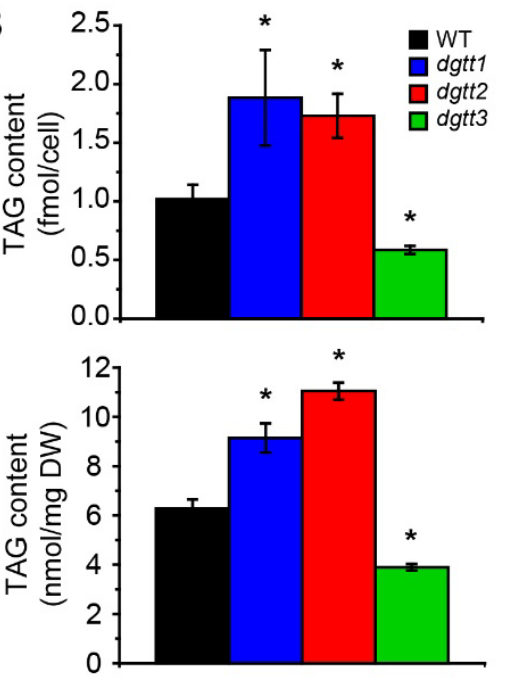

DGTT2
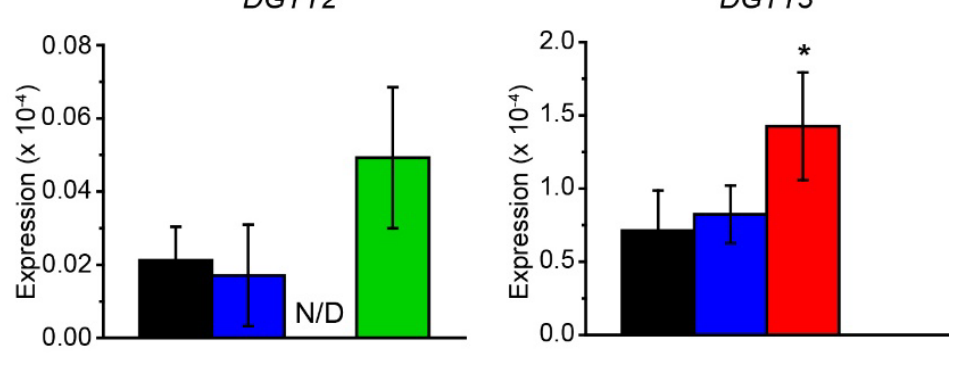

Fig 1. Genetic structure, phenotype and gene expression of the $d g t t 1$, dgtt2, dgtt3 knockout mutants obtained from the CLiP library. (A) Locations of the insertion sites of the CIB1 cassette (brown triangles) and of the primer pairs (black arrows) used to detect gene expression in the DGTT1, DGTT2 and DGTT3 genes are presented. (B) TAG content of the $d g t t 1$ (blue), dgtt2 (red), dgtt3 (green) mutants and the parent strain CC5325 (black, WT) grown was measured at $72 \mathrm{~h}$ under P deprivation. (C) Relative transcriptional level of selected TAG biosynthesis genes (DGTT1, DGTT2, DGTT3, PDAT and PGD1) in the dgtt1 (blue), dgtt2 (red), dgtt3 (green) mutants and the parent strain CC5325 (black) was measured at $24 \mathrm{~h}$ under P deprivation. Transcripts of DGTT1 in the dgtt1 mutant and DGTT2 in the dgtt2 mutant were nondetectable (N/D). Data represents mean \pm standard deviation (SD) from three biological repeats. An asterisk indicates statistical significance by Student's $t$-test $(p$ value $\leq 0.05$ ) when compared to WT. 


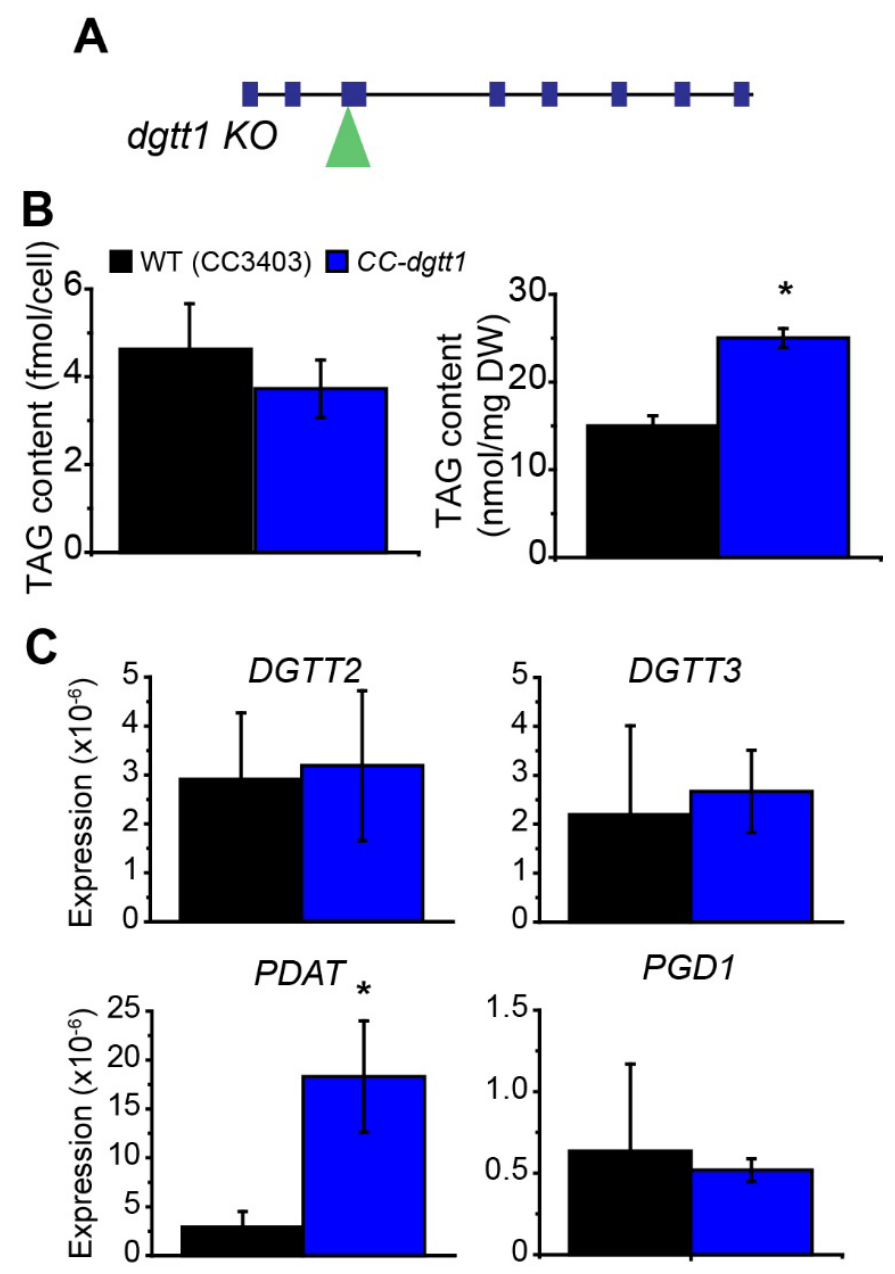

Fig 2. Genetic structure, phenotype and gene expression of the CRISPR-derived dgtt1 knockout mutant. (A) The location of an AphIII cassette insertion (green triangle) generated by CRISPR in the DGTTI gene are presented. (B) TAG content of the $C C$ - $d g t t 1$ mutant (blue) and the parent strain CC3403 (black, WT) grown was measured at $72 \mathrm{~h}$ under $\mathrm{P}$ deprivation. (C) Relative transcriptional level of selected TAG biosynthesis genes (DGTT2, DGTT3, PDAT and PGD1) in the dgttl mutant (blue) and the parent strain CC3403 (black) was measured at $48 \mathrm{~h}$ under P deprivation. Data represents mean \pm standard deviation (SD) from three biological repeats. An asterisk indicates statistical significance by Student's $t$-test ( $p$ value $\leq 0.05$ ). 


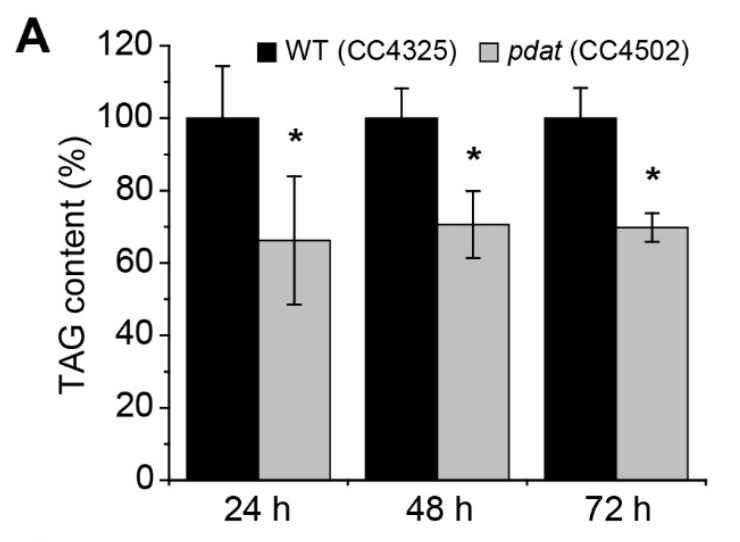

B
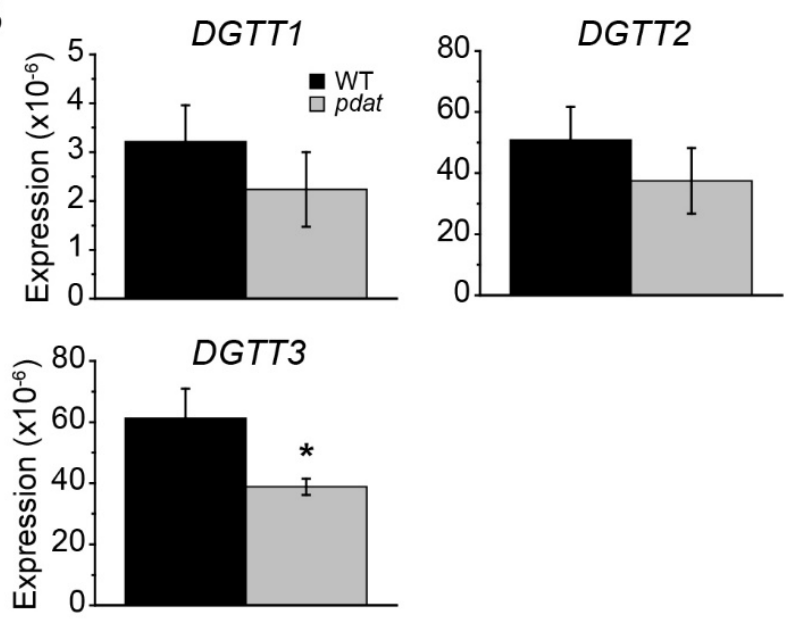

Fig 3. Phenotype and gene expression of the pdat mutant (CC4502). (A) Relative concentrations of TAG from the pdat knockout strain CC4502 (gray) and the wild-type CC4425 (black, WT) grown under 24 h-, 48 h-, 72 h- and 96 h-P deprivation was shown. (B) Relative transcriptional level of selected TAG biosynthesis genes (DGTT1, DGTT2, DGTT3) in the pdat mutant (gray) and the wild-type (black) was measured at $24 \mathrm{~h}$ under $\mathrm{P}$ deprivation. Data represents mean \pm standard deviation (SD) from three biological repeats. An asterisk indicates statistical significance by Student's $t$-test ( $p$ value $\leq 0.05$ ). 
A

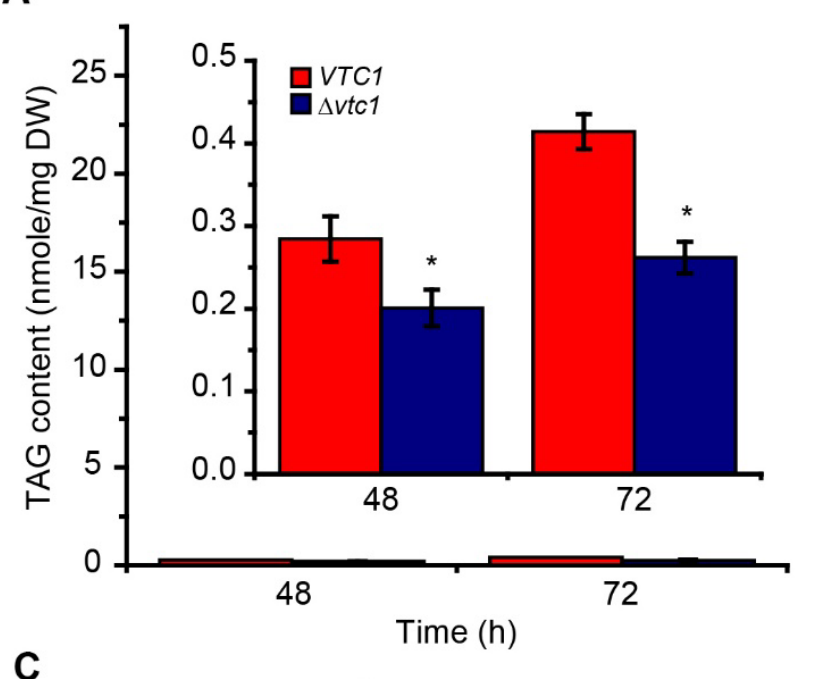

B

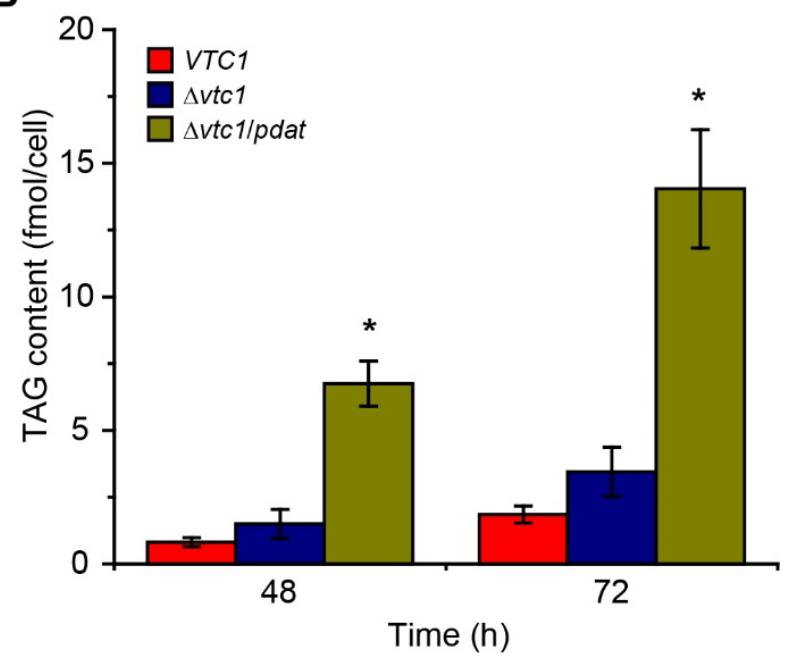

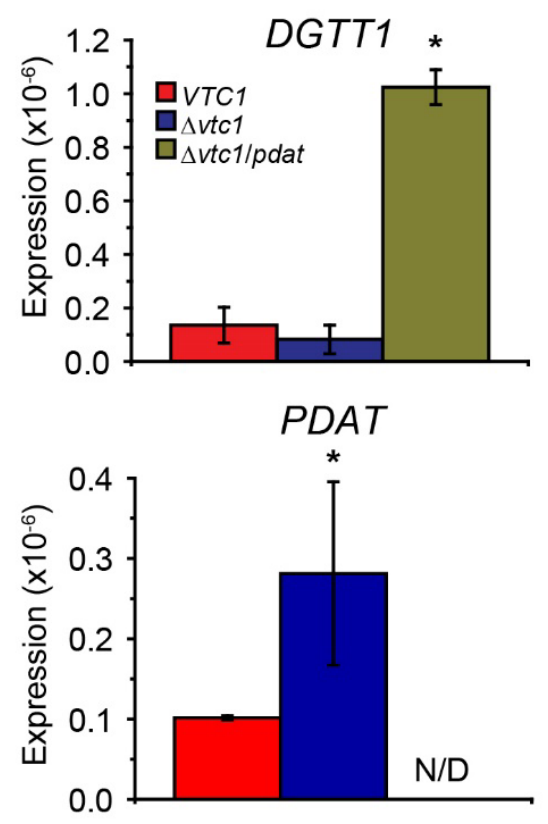
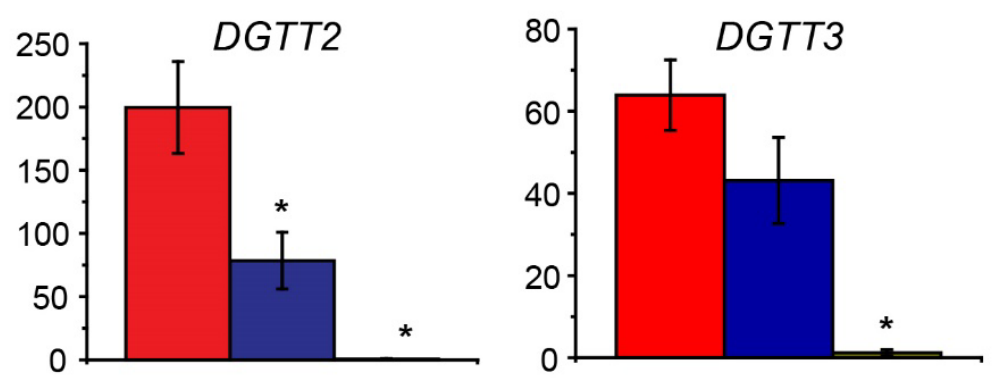

PGD1

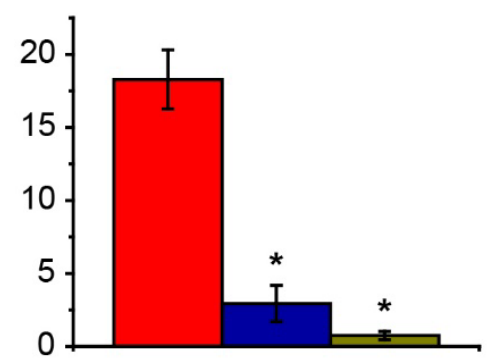

Fig 4. Phenotype and gene expression of the VTC1 strain, $\Delta v t c 1$ single mutant and $\Delta v t c 1 / p d a t$ double mutant. (A) TAG content of the $\triangle v t c 1$ strain CC5165 and the VTC1 rescue strain CC5166 grown was measured at $48 \mathrm{~h}$ and $72 \mathrm{~h}$ under P replete. (B) TAG content of the VTC1 strain, $\Delta v t c 1$ strain and the $\Delta v t c 1 / p d a t$ strain grown was measured at $48 \mathrm{~h}$ and $72 \mathrm{~h}$ under $\mathrm{P}$ deprivation. (C) Relative transcriptional level of selected genes (DGTT1, DGTT2, DGTT3, PDAT and PGD1) was measured at $24 \mathrm{~h}$ under P deprivation. Transcripts of $P D A T$ in the $\triangle v t c 1 / p d a t$ double mutant were nondetectable (N/D). Data represents mean \pm standard deviation (SD) from three biological repeats. An asterisk indicates statistical significance by Student's $t$-test ( $p$ value $\leq 0.05$ ). 